\title{
Piezomagnetic behavior: experimental observations and multiscale modeling
}

\author{
Achraf Ouaddi ${ }^{1,2}$, Olivier Hubert ${ }^{1, *}$, Jader Furtado ${ }^{2}$, Daniel Gary ${ }^{2}$, and Sophie Depeyre ${ }^{3}$ \\ ${ }^{1}$ Université Paris-Saclay, ENS Paris-Saclay, CNRS, LMT, 61 avenue du Président Wilson, 94235 Cachan, France \\ 2 Air-liquide R\&D, Innovation Campus Paris, 1 chemin de la Porte des Loges, BP126, Les Loges-en-Josas, 78350 \\ Jouy-en-Josas Cedex, France \\ ${ }^{3}$ Léonard de Vinci Pôle Universitaire, 92916 Paris La Défense Cedex, France
}

Received: 17 October 2019 / Accepted: 29 April 2020

\begin{abstract}
This work deals with the study of magnetoelastic coupling in the framework of non-destructive testing. Experimental hysteretic and cyclic piezomagnetic measurements carried out on a dual-phase steel submitted to different magnetic field and stress conditions are reported. The effect of concomitant magnetic field and stress, considering static or variable amplitudes, is discussed. A new multiscale modeling of piezomagnetic hysteresis is finally proposed.
\end{abstract}

Keywords: magnetic and piezomagnetic hysteresis / magnetoelastic coupling / multiscale modeling

\section{Introduction}

The submission of a ferromagnetic material to a magnetic field leads to a geometric distortion of the sample. This deformation is called the magnetostriction strain. Conversely, the application of an external mechanical stress to a ferromagnetic material, initially magnetized even faintly, leads to a significant change of magnetization. These behaviors are symptomatic of the same coupling called the piezomagnetic or Villari effect [1]. Indeed mechanical stress is one of the three major factors that can affect the magnetization in addition to magnetic field and temperature. The magnetic behavior is thus sensitive to any mechanical loading and its evolution will be different depending on the loading level, direction and nature [2]. Since then, numerous works have been initiated to understand, model and use this coupling effect in industrial applications such as on Non-Destructive Testing (NDT). We can cite for example: magnetic particle inspection (MPI), Eddy currents, Barkhausen noise, etc. The purpose of all these magnetic methods is to extract information about the metallurgical and mechanical states of a material by analyzing the electromagnetic signal [3].

Piezomagnetic control methods have undergone recent developments $[4,5]$ but still face modeling issues, especially when no controlled magnetic field is applied [6]. The development, the implementation and the generalization of such techniques involve, in one hand, the establishment of experiments to highlight the magnetoelastic coupling in a controlled framework, and on the other

\footnotetext{
* e-mail: olivier .hubert@ens-paris-saclay.fr
}

hand, the development of innovative modeling methods. Both aspects are addressed in this paper. The measurements presented are related to the hysteretic and cyclic piezomagnetic behavior [7] of a dual-phase steel submitted to various magnetic field and stress conditions. After describing a multiscale approach of magneto-elastic couplings in a reversible framework and of associated magnetic hysteresis model, we then propose a new modeling of piezomagnetic hysteresis behavior.

\section{Material and procedure}

\subsection{Material}

The material used for the experiments is a dual-phase low carbon steel (DPS780 from ArcelorMittal). Its microstructure consists of about 30\% vol. of hard (mechanically) martensite islands dispersed in a soft (ductile) ferritic matrix. From a magnetic consideration the material can be considered as 70\%-pure iron as a first approximation since martensite exhibits a susceptibility much lower than the susceptibility of ferrite. Its contribution is neglected for the modeling. This material has been the subject of several studies in particular in the context of the link between mechanical state and magnetic behavior $[8,9]$.

\subsection{Set up and experiments}

Samples consist in $200 \mathrm{~mm}$ long, $12.5 \mathrm{~mm}$ wide and $1.6 \mathrm{~mm}$ thick strips (Fig. 1) suitable for the experimental set-up used for experiments. 


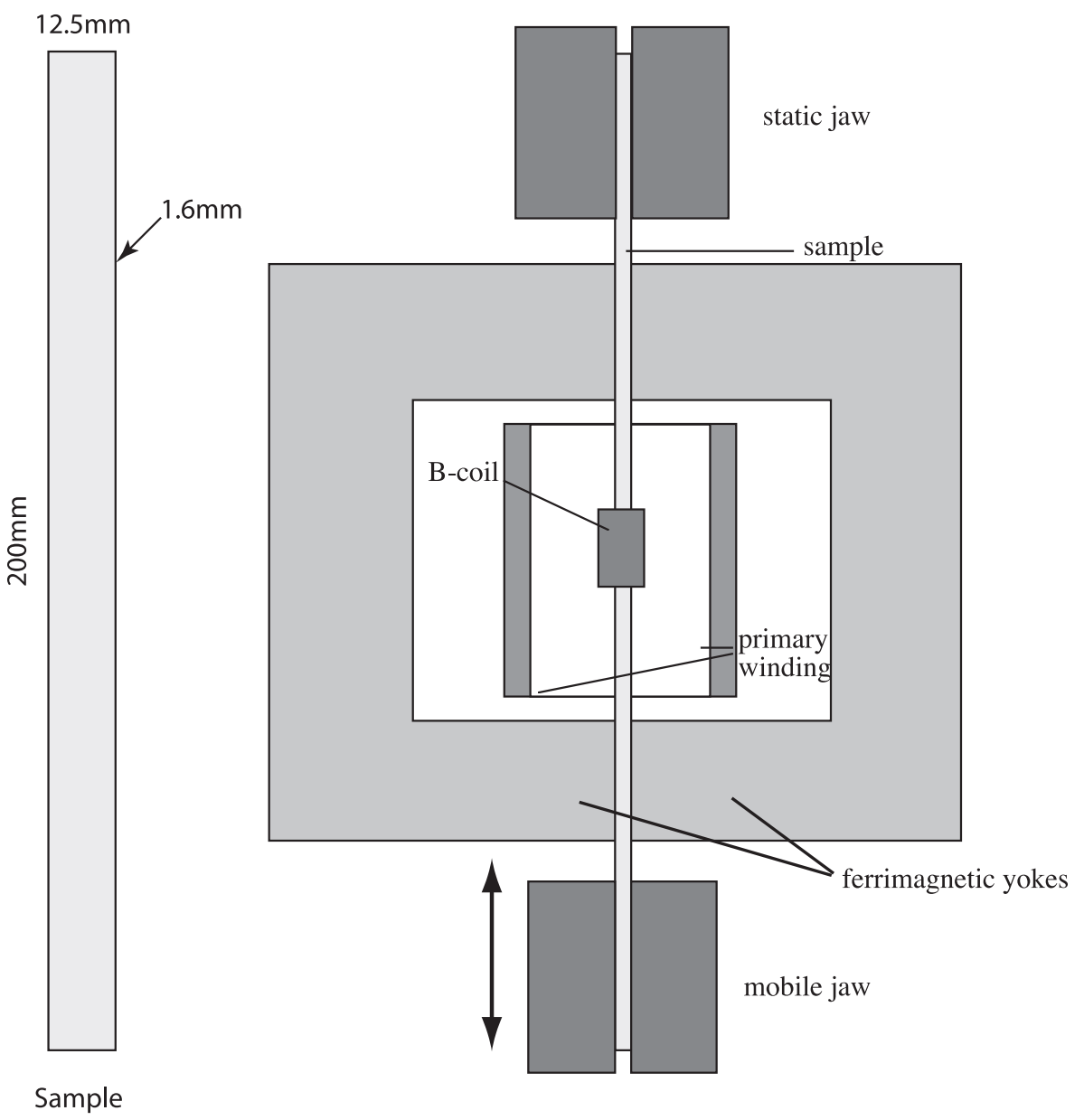

Fig. 1. Sample geometry and experimental set up.

Two different experiments have been carried out in this study: hysteretic measurements under static mechanical stress and piezomagnetic measurements under cyclic mechanical stress. For both tests, the same experimental apparatus has been used as sketched in Figure 1 (details concerning the experimental apparatus can be found in $[4,5])$. The sample is placed between two " $U$ " ferrite yokes allowing the re-close of the magnetic flux. The excitation field is applied by the primary winding formed of $N=81$ turns and fed by an electrical current of intensity $i(t)$. The magnetic flux in the sample is estimated by integrating the induced voltage at the terminals of a B-coil, formed of $n=700$ turns. The measurement of the magnetic flux in the sample allows the induction $B$, then the magnetization $M$ to be calculated. The setup is mounted inside an electro-hydraulic MTS machine, applying from compressive to tensile stresses. The setup allows the study of magnetic behavior of the material under stress. A LabView system allows the simultaneous acquisition and recording of the different quantities of interest (force, displacement, applied current and induced voltage).

The first part of the work consists in measuring the magnetic hysteresis behavior of the material under static uniaxial stress. The magnetic cycles are produced by applying a triangular current intensity (allowing a constant magnetic field rate). Test parameters are the mean magnetic field $\left(H_{m}=0 \mathrm{~A} / \mathrm{m}\right)$, the frequency $f_{\text {mag }}(0.1 \mathrm{~Hz})$, the magnetic amplitude $\Delta H(850 \mathrm{~A} / \mathrm{m}$, $1700 \mathrm{~A} / \mathrm{m}, 3400 \mathrm{~A} / \mathrm{m}, 8500 \mathrm{~A} / \mathrm{m}, 15000 \mathrm{~A} / \mathrm{m})$ and the static stress value $\sigma_{\text {stat }}$ (from $-100 \mathrm{MPa}$ to $300 \mathrm{MPa}$ ). The procedure is composed of the following steps: (1) application of the mechanical loading $\sigma_{\text {stat }} ;(2)$ demagnetizating by applying a decreasing amplitude sinusoidal current; (3) application of magnetic field $H(t)$ and measurement of induced voltage; (4) recording of measurements.

The second part of this work consists to measure the piezomagnetic behavior of the material under different levels of the static magnetic field $H_{\text {stat }}(850 \mathrm{~A} / \mathrm{m}$, $1700 \mathrm{~A} / \mathrm{m}, 3400 \mathrm{~A} / \mathrm{m}, 8500 \mathrm{~A} / \mathrm{m}, 15000 \mathrm{~A} / \mathrm{m})$. A sinusoidal stress waveform has been used for mechanical loading. Test parameters are the mean stress $\sigma_{m}$ (from $-50 \mathrm{MPa}$ to $300 \mathrm{MPa}$ ), the stress amplitude $\Delta \sigma$ (from $50 \mathrm{MPa}$ to $200 \mathrm{MPa}$ ) and the frequency of loading $f_{\mathrm{mec}}$ $(0.5 \mathrm{~Hz})$. The mechanical loading is chosen in the range $[-100 \mathrm{MPa} 300 \mathrm{MPa}]$ in order to avoid the sample yielding (yield stress $\sigma_{y}=425 \mathrm{MPa}$ ) and buckling. ${ }^{1}$

\footnotetext{
${ }^{1}$ The critical buckling stress is given by the Euler formula. Considering an unsupported length of $80 \mathrm{~mm}$, and a Young modulus of $210 \mathrm{GPa}$, the critical buckling stress is estimated to $-280 \mathrm{MPa}$.
} 
The piezomagnetic measurement procedure is composed of the following steps: (1) cyclic mechanical loading of amplitude $\Delta \sigma ;(2)$ application of the static magnetic field after demagnetizing; (3) measurement and averaging over 50 cycles after stabilization of induced voltage; (4) recording of the measurements.

\section{Experimental results}

\subsection{Magnetic hysteresis}

When the material is submitted to an alternative magnetic field, the magnetization forms an hysteresis loop illustrating the irreversibility of the magnetic behavior and the presence of dissipative phenomena. Figure 2 depicts the evolution of the magnetic hysteresis loop at different magnetic field levels for $\sigma_{\text {stat }}=0 \mathrm{MPa}$. The cycles are nested inside each other. It can be noticed that the evolution of the shape of the hysteresis cycles is highly dependent on the magnetic field level. Indeed, we can see that the maximal $d M / d H$ slope and the cycle area increase with increasing amplitude of the magnetic field. It can be noticed also that magnetization saturation is not far to be reached for loop obtained at the highest magnetic field strength. Its level remains however lower than the theoretical saturation of pure iron $\left(1.71 \times 10^{6} \mathrm{~A} / \mathrm{m}\right)$ due to the martensite ratio and various demagnetizing effects.

Magnetic measurements reported in Figure 3 represent the hysteretic cycles under uniaxial stress. Measurements lead to conventional results for low carbon steel: global degradation of magnetization due to compressive stress at a given magnetic field (decrease of magnetic susceptibility), improvement due to tensile stress and Villari reversal effect [9]. The Villari reversal is associated with the change of $d M /\left.d \sigma\right|_{H}$ sign: at weak and intermediate magnetic field, the ratio $d M /\left.d \sigma\right|_{H}$ is positive. It becomes negative at higher magnetic field leading to a change of sign of $d M /\left.d \sigma\right|_{H}$ (becoming negative and corresponding to the reversal point). A higher stress level progressively shifts the Villari reversal point to the lower magnetic field values. It can be noticed that the Villari reversal can be related to the change of sign of the magnetostriction vs. magnetic field behavior [10].

\subsection{Piezomagnetic hysteresis}

Figure 4 shows the magnetic response associated with the variation of stress $\left(\sigma_{m}=100 \mathrm{MPa}, \Delta \sigma=200 \mathrm{MPa}\right.$, $\left.\mathrm{f}_{\text {mec }}=0.5 \mathrm{~Hz}\right)$ for $H_{\text {stat }}=(850 \mathrm{~A} / \mathrm{m}, 1700 \mathrm{~A} / \mathrm{m}, 3400 \mathrm{~A} / \mathrm{m}$, $8500 \mathrm{~A} / \mathrm{m}, 15000 \mathrm{~A} / \mathrm{m})$. In order to facilitate the comparison of the experimental and numerical results, we considered the magnetic state at $300 \mathrm{MPa}$ as a reference state for magnetization. As shown in Figure 4, the piezomagnetic cycles are not symmetric and non-monotonous: positive stress leads to quasi-reversible situation and negative stress increases the cycle area. Again, for a weak to intermediate magnetic field, instantaneous slope $d M /\left.d \sigma\right|_{H}$ is practically positive for compressive to weak tensile stress. The sign reversal of $d M /\left.d \sigma\right|_{H}$ occurs for

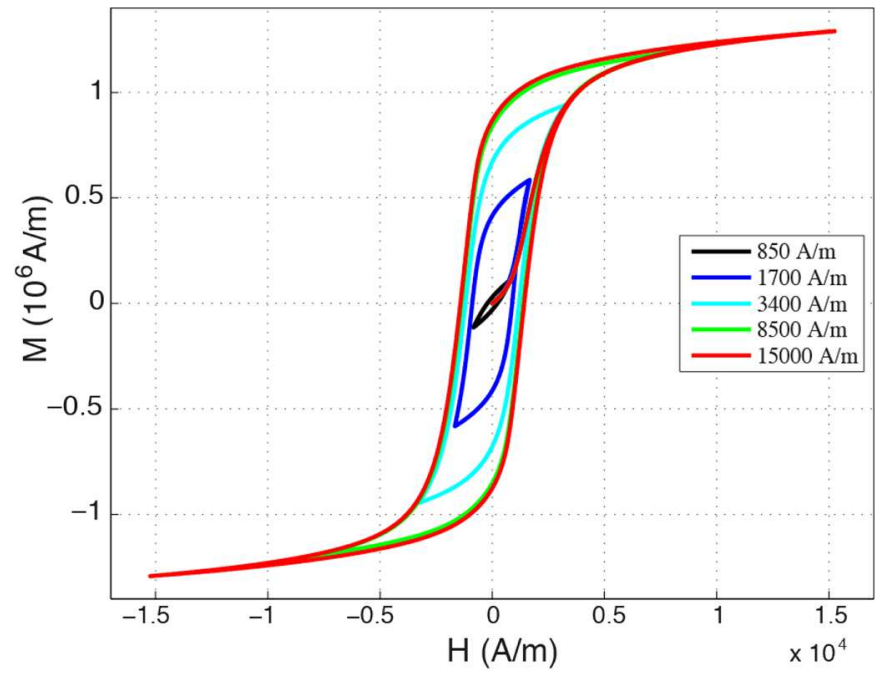

Fig. 2. Experimental results: magnetic hysteresis at increasing magnetic field levels without applied stress $-M=f(H, \sigma=$ $0 \mathrm{MPa})$.

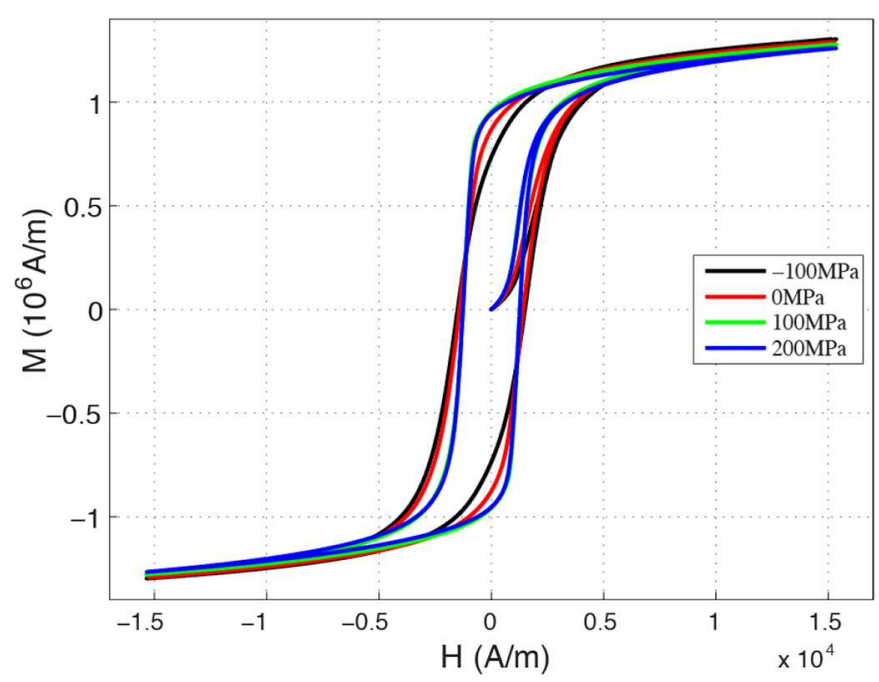

Fig. 3. Experimental results: hysteretic cycles under different mechanical loading conditions $-M=f(H, \sigma=$ cte $)$.

stress levels varying from $70 \mathrm{MPa}$ to $230 \mathrm{MPa}$ depending on position on the cycle. At higher magnetic field strength, reversal clearly occurs at lower stress. High magnetic fields $(\mathrm{H} \geq 4 \mathrm{k} \mathrm{A} / \mathrm{m})$ lead to a negative and quasi constant slope. Moreover the piezomagnetic behavior becomes quasi-reversible. This result is interesting if a sensor application is looked for. Indeed, the applied mechanical stress could be estimated by only measuring the magnetization of material (and knowing a reference point).

Readers interested in magnetostriction behavior of this material under stress and associated models are invited to consult reference [9]. 


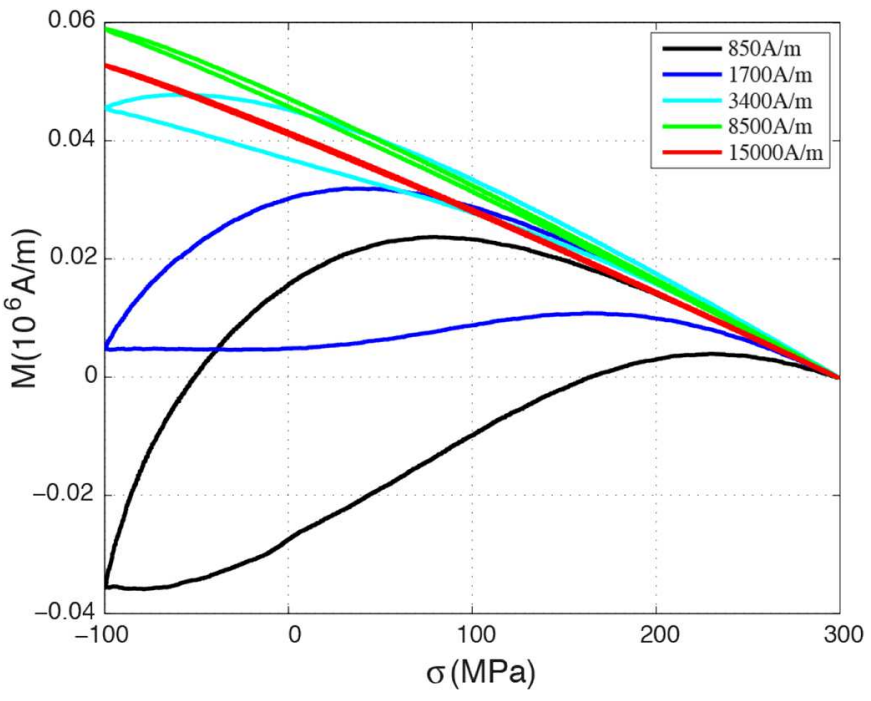

Fig. 4. Experimental results: piezomagnetic behavior obtained under cyclic stress conditions and at different magnetic field levels $-M=f(H=$ cte, $\sigma)$.

\section{Multiscale modeling}

The model is derived from a description of reversible magneto-elastic behavior $[10,11]$ extended recently to magnetic hysteresis [12]. This description relies on the definition of the material Gibbs free energy at the magnetic domain scale and the estimation of the domains volume fractions using a at equilibrium stochastic approach at the grain scale. Some scale transition rules are used to define the behavior at the polycrystalline scale considered as the representative volume element (RVE).

\subsection{Gibbs free energy and reversible modeling}

The Gibbs free energy density is written at the magnetic domain scale $\alpha$. The reader can find in [10] complete and comprehensive explanation and hypotheses about the construct of this energy functional. Indeed at the domain scale, both magnetization $\overrightarrow{\mathbf{M}}_{\alpha}$ and magnetostriction strain $\varepsilon_{\alpha}^{\mu}$ (seen as a free deformation tensor) can be considered as homogeneous. The norm of $\overrightarrow{\mathbf{M}}_{\alpha}$ is the saturation magnetization $M_{s}$ as a first constant of the model (see Eq. (1), where $\gamma_{\alpha}^{k}$ are the direction cosines of magnetization vector and $\overrightarrow{\mathbf{e}}_{\alpha}^{k}$ are the unit vectors of the crystallographic frame). In the framework of linear magnetoelastic coupling, $\varepsilon_{\alpha}^{\mu}$ is stress independent and isochorus, given by equation (2), where $\lambda_{100}$ and $\lambda_{111}$ are two other constants measuring the saturation deformation along $\langle 100\rangle$ and $\langle 111\rangle$ crystallographic directions respectively. Moreover in the framework of this paper and for the seek of simplicity, stress $\boldsymbol{\sigma}$ and magnetic field $\overrightarrow{\mathbf{H}}$ are considered homogeneous over the RVE (avoiding any complex rules for localization or homogenization). Appendix A details how it is possible to implement other estimates (homogeneous strain or self-consistent schemes) from grain to RVE transitions. Piezomagnetic modeling results are however close together whatever the estimate.

$$
\begin{gathered}
\overrightarrow{\mathbf{M}}_{\alpha}=M_{s} \gamma_{\alpha}^{k} \cdot \overrightarrow{\mathbf{e}}_{\alpha}^{k} \\
\varepsilon_{\alpha}^{\mu}=\frac{3}{2}\left(\begin{array}{ccc}
\lambda_{100}\left(\gamma_{1}^{2}-\frac{1}{3}\right) & \lambda_{111} \gamma_{1} \gamma_{2} & \lambda_{111} \gamma_{1} \gamma_{3} \\
\lambda_{111} \gamma_{1} \gamma_{2} & \lambda_{100}\left(\gamma_{2}^{2}-\frac{1}{3}\right) & \lambda_{111} \gamma_{2} \gamma_{3} \\
\lambda_{111} \gamma_{1} \gamma_{3} & \lambda_{111} \gamma_{2} \gamma_{3} & \lambda_{100}\left(\gamma_{3}^{2}-\frac{1}{3}\right)
\end{array}\right) .
\end{gathered}
$$

Following these simplifications, the Gibbs free energy density at the magnetic domain scale is simply expressed as:

$$
\begin{aligned}
g_{\alpha}(\overrightarrow{\mathbf{H}}, \boldsymbol{\sigma})= & K_{1}\left(\gamma_{1}^{2} \gamma_{2}^{2}+\gamma_{2}^{2} \gamma_{3}^{2}+\gamma_{3}^{2} \gamma_{1}^{2}\right)-\mu_{0} \overrightarrow{\mathbf{H}} \cdot \overrightarrow{\mathbf{M}}_{\alpha} \\
& -\frac{1}{2} \boldsymbol{\sigma}: \mathbb{C}^{-1}: \boldsymbol{\sigma}-\boldsymbol{\sigma}: \varepsilon_{\alpha}^{\mu},
\end{aligned}
$$

where $\mu_{0}$ is the vacuum magnetic permeability and $\mathbb{C}$ the stiffness tensor of the medium.

The term $K_{1}\left(\gamma_{1}^{2} \gamma_{2}^{2}+\gamma_{2}^{2} \gamma_{3}^{2}+\gamma_{1}^{2} \gamma_{3}^{2}\right)$ is the magnetocrystalline energy density with $K_{1}$ a magnetocrystalline constant. This non-convex term allows the so-called easy magnetization directions (six $\langle 100\rangle$ directions for iron and iron alloys) to be defined. These directions define the number of domains (or domain families $\alpha$ ) that can be seen as crystallographic variants. Calculation of magnetic domains volume fraction is made possible by using a at equilibrium stochastic approach, which neglects the transition zones (domain walls) between domains. This calculation is complemented by a minimization process of $g_{\alpha}$ regarding the direction cosines of domain families $\alpha$ to take the so-called magnetization rotation mechanism into account. Following this strategy, a Boltzmann function gives the solution of the stochastic approach [13] (with $V_{0}$ a reference microscopic volume, $k_{B}$ the Boltzmann constant $\left(1.38 \times 10^{-23} \mathrm{~J} \mathrm{~K}^{-1}\right)$ and $T$ the temperature $(293 \mathrm{~K}))$.

$$
\begin{gathered}
f_{\alpha}=\frac{\exp \left(-\frac{V_{0}}{k_{B} T} g_{\alpha}\right)}{\sum_{i=1}^{6} \exp \left(-\frac{V_{0}}{k_{B} T} g_{i}\right)} \\
\overrightarrow{\boldsymbol{\gamma}}_{\alpha}=\min \left(g_{\alpha}(\overrightarrow{\boldsymbol{\gamma}}, \overrightarrow{\mathbf{H}}, \boldsymbol{\sigma})\right) .
\end{gathered}
$$

It must be noticed that since the mechanical contribution $\frac{1}{2} \sigma: \mathbb{C}^{-1}: \boldsymbol{\sigma}$ is uniform over the grain, it does not participate to the energy equilibrium.

Averaging operations end the process as expressed in equations (6) and (7), with $N_{g}$ the number of grains $g$ involved in the process (a discrete orientation distribution function - ODF - extracted from Electron Back -Scattered Diffraction - EBSD - measurement is used).

$$
\begin{aligned}
\overrightarrow{\mathbf{M}} & =\frac{1}{N_{g}} \sum_{g}\left(\sum_{\alpha=1}^{6} f_{\alpha} \overrightarrow{\mathbf{M}}_{\alpha}\right) \\
\varepsilon^{\mu} & =\frac{1}{N_{g}} \sum_{g}\left(\sum_{\alpha=1}^{6} f_{\alpha} \varepsilon_{\alpha}^{\mu}\right) .
\end{aligned}
$$


Both stress and magnetic field variations are the possible loadings of the problem. A cyclic magnetic field at constant stress leads to magnetic and field-induced magnetostriction behaviors at constant stress. A cyclic stress at constant magnetic field leads to the piezomagnetic and stress-induced magnetostriction behaviors at constant magnetic field.

\subsection{Irreversible modeling - application of Hauser's modeling to Piezomagnetic cycle}

The multiscale approach is by definition anhysteretic, meaning that it is restricted to the reversible part of magneto-elastic behavior. It has been proposed in [12] to introduce hysteresis effects in the multiscale model in terms of irreversible magnetic field. The dissipation is introduced (initially in the single crystal model) by adding an irreversible contribution $H_{\text {irr }}$ to the anhysteretic magnetic field $H$ (considered as the reversible magnetic field $\left.H_{\text {rev }}\right)$. The definition of $H_{\text {irr }}$ is based on the works by Hauser [14], extended to magneto-mechanical loadings.

The Hauser's model predicts the magnetic state of the ferromagnetic material by minimizing the overall energy state of the system. This approach is based on a physical and probabilistic description of the volume distribution of the magnetic domain families. The dissipative behavior results from the probability of encountering a defect during the displacement of magnetic walls separating the magnetic domains. The expression of the irreversible energy is a decreasing exponential function which depends on the variations of the anhysteretic volume fraction. It reflects the fact that a wall has a lower probability of being pinned to a defect, when it is away from it. Hauser has often simplified this development by studying a load applied along an easy magnetization axis of the material and considering quantities as isotropic and at the macroscopic scale. Following these simplifications, equation (8) gives the expression of $H_{\text {irr }}$ retained for this work.

$$
H_{\mathrm{irr}}=\delta\left(H_{c}+a\left|H_{\mathrm{rev}}\right|\right)\left[1-\kappa \exp \left(-\frac{k_{a}}{\kappa}\left|M-M_{\mathrm{prev}}\right|\right)\right]
$$

where

$$
\kappa=2-\kappa_{0} \exp \left(-\frac{k_{a}}{\kappa_{0}}\left|M-M_{\mathrm{prev}}\right|\right) .
$$

$H_{\text {irr }}$ is assumed to be parallel to $H$ and $H_{\text {rev }}$, since $H=H_{\mathrm{rev}}+H_{\mathrm{irr}}$. Hysteresis is restricted to magnetic field amplitude effects, rotational effects cannot be modeled. $\delta$ is equal to \pm 1 , depending on the loading direction. The sign of $\delta$ starts as positive and is then changed at each inversion in loading direction. $H_{c}$ denotes the coercive field of the material (magnetic field required to annul the remnant magnetization for a major cycle). $a, k_{a}$ and $\kappa$ are material parameters. $a$ controls the first magnetization behavior, and $k_{a}$ and $\kappa$ the width and inclination of the hysteresis cycle. The value of $\kappa$ changes each time there is an inversion in the loading direction. The new $\kappa$ value is calculated from the previous value $\kappa_{0}$ according to equation (9). The initial $\kappa$ value is a material constant. $M_{\text {prev }}$ is the value of magnetization $M$ at the previous inversion of the loading direction. In the case of a purely magnetic loading, an inversion of loading direction is defined as a change of sign for the time derivative of the applied magnetic field.

Extension of this model to stress loading is key once a piezomagnetic cycle is expected to be modeled. It must be recalled that the expression from Hauser comes from an observation and calculation of the energy dissipated by the pinning mechanisms and by the wall jumps. It depends only on the path made by the walls and does not depend on the whether the walls movement is generated by the application of a magnetic field or a mechanical stress. Indeed the intrinsic dissipation part of Clausius-Duhem inequality can be built at the macroscale using irreversible magnetic field or irreversible stress following:

$$
\mu_{0} \dot{\overrightarrow{\mathbf{M}}} \cdot \overrightarrow{\mathbf{H}}_{\mathrm{irr}}+\dot{\boldsymbol{\varepsilon}}^{\mu} \cdot \sigma_{\mathrm{irr}} \geq 0 .
$$

It is possible to propose a dissipation pseudo-potential $\Phi$ (convex and positive) so that, for a step-time $d t$ and considering a monotonous evolution of magnetization (by part) in uniaxial condition, one gets:

$$
\overrightarrow{\mathbf{H}}_{\text {irr }}=\frac{d \Phi}{\mu_{0} d \overrightarrow{\mathbf{M}}} d t
$$

The definition of $H_{\text {irr }}$ given by Hauser allows an appropriate definition of dissipation pseudo-potential $\Phi$. The same comment can be made for irreversible stress. Considering a monotonous evolution of magnetostriction deformation in uniaxial condition, one gets:

$$
\sigma_{\mathrm{irr}}=\frac{d \Phi}{d \varepsilon^{\mu}} d t
$$

The definition of an irreversible stress directly inspired from the irreversible magnetic field definition can be proposed, restricted to 1D loading (only axial stress and strain components are considered):

$$
\sigma=\sigma_{\text {rev }}+\sigma_{\text {irr }}
$$

with $\sigma_{\text {rev }}$ the reversible stress leading to the at equilibrium magnetization using the multiscale model. $\sigma_{\text {irr }}$ is given by:

$$
\sigma_{\text {irr }}=\delta\left(\sigma_{C}+a^{\prime}\left|\sigma_{\text {rev }}\right|\right)\left[1-\kappa^{\prime} \exp \left(-\frac{k_{a}^{\prime}}{\kappa^{\prime}}\left|\varepsilon^{\mu}-\varepsilon_{\text {prev }}^{\mu}\right|\right)\right]
$$

and

$$
\kappa^{\prime}=2-\kappa_{0}^{\prime} \exp \left(-\frac{k_{a}^{\prime}}{\kappa_{0}^{\prime}}\left|\varepsilon^{\mu}-\varepsilon_{\text {prev }}^{\mu}\right|\right)
$$

These equations define four more material coefficients: a coercive stress $\sigma_{c}$, and parameters $a^{\prime}, k_{a}^{\prime}$ and $\kappa_{0}^{\prime} . a^{\prime}$ controls the first stress-induced magnetostriction behavior, and $k_{a}^{\prime}$ and $\kappa^{\prime}$ the width and inclination of the stressinduced magnetostriction hysteresis cycle. An inversion of loading direction (leading to a change of the sign of 

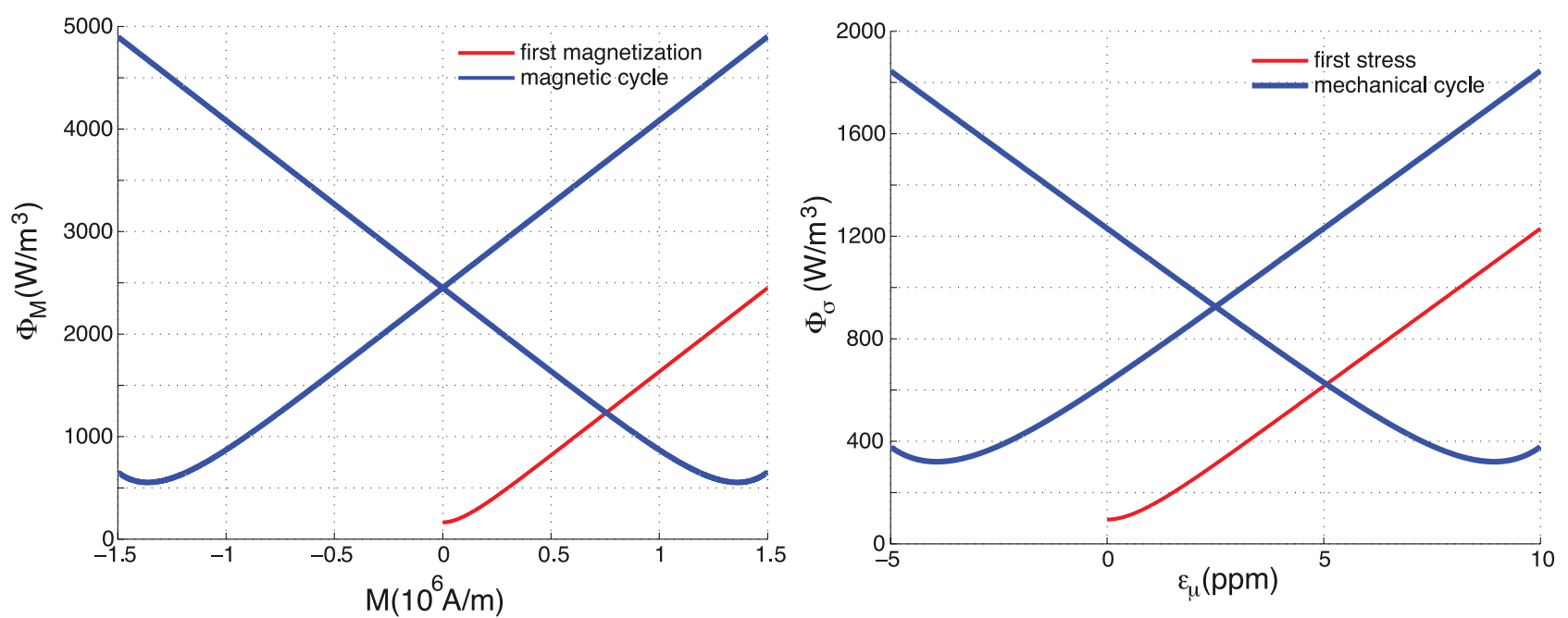

Fig. 5. Illustration of dissipation pseudo-potential for magnetization $\Phi_{M}$ at constant stress and for stress $\Phi_{\sigma}$ at constant magnetic field during the transient first loading and the (stabilized) magnetic and mechanical cycles.

parameter $\delta$ ) is defined as a change of sign for the time derivative of the applied stress. Figure 5 illustrates the dissipation pseudo-potential for magnetization $\Phi_{M}$ at constant stress and for stress $\Phi_{\sigma}$ at constant magnetic field during the transient first loading (first magnetization and first stress) and cycles (one curve per branch) for the parameters reported in Tables 1 and 2 (but considering parameters $a$ and $a^{\prime}$ at zero). It exhibits the appropriate properties (positive and convex).

However, it must be underlined that the stress-induced magnetostriction cycle (stress vs magnetostriction) can hardly be obtained experimentally. The more relevant indicator of the effect of a variable stress is of course the piezomagnetic cycle. Comparisons of experimental and modeled piezomagnetic cycles are possible thanks to the definition of irreversible stress. They can help to adjust the modeling parameters. It must be underlined on the other hand that following the previous remarks and discussion, a piezomagnetic cycle is not representative of an energy dissipation, unlike a magnetostriction vs. stress cycle.

\section{Modeling results and discussion}

\subsection{Numerical parameters used for modeling}

Physical constants of pure iron have been used for the anhysteretic parameters. They are reported in Table 1 . An orientation data file made of 440 orientations has been used to model the RVE. Related pole figures are plotted in Figure 6. Table 2 gathers parameters used to model the irreversible magnetic field and stress, that fit properly the experimental results, but without numerical optimization (improvements are possible).

\subsection{Magnetic hysteresis}

In this section the multiscale model is applied to describe the magnetic hysteresis behavior of the dual-phase steel. As a first step, the material is supposed to have the same
Table 1. Parameters used in the multiscale modeling anhysteretic behavior.

\begin{tabular}{lllll}
\hline Parameter & $\mathrm{M}_{s}$ & $K_{1}$ & $\lambda_{100} ; \lambda_{111}$ & $V_{0}$ \\
\hline Value & $1.71 \times 10^{6}$ & 48 & $21.5 ;-21.5$ & 8000 \\
Unit & $\mathrm{A} / \mathrm{m}$ & $\mathrm{kJ} / \mathrm{m}^{3}$ & $\mathrm{ppm}$ & $\mathrm{nm}^{3}$ \\
\hline
\end{tabular}

Table 2. Parameters used in the multiscale modeling cyclic magnetic and cyclic piezomagnetic behaviors.

\begin{tabular}{lllllllll}
\hline Parameter & $H_{c}$ & $a$ & $k_{a}$ & $\kappa_{0}$ & $\sigma_{c}$ & $a^{\prime}$ & $k_{a}^{\prime}$ & $\kappa_{0}^{\prime}$ \\
\hline Value & 1300 & 0.0 & $1 \times 10^{-5}$ & 1 & 123 & 0.0 & $1.3 \times 10^{6}$ & 1 \\
Unit & $\mathrm{A} / \mathrm{m}$ & - & $\mathrm{m} . \mathrm{A}^{-1}$ & - & $\mathrm{MPa}$ & - & - & - \\
\hline
\end{tabular}

magnetic behavior as pure iron. Figure 7 reports the hysteretic cycles at the unloaded state for different magnetic field levels used for experiments (Fig. 2). In the other hand, the major hysteresis loops at the unloaded state and under $-100 \mathrm{MPa}, 100 \mathrm{MPa}$ and $200 \mathrm{MPa}$ are plotted in Figure 8 (to be compared with results plotted in Fig. 3). The general trends are correctly met. The effect of tensile and compressive uniaxial stress on the material magnetization are properly rendered. Both experimental and numerical results show that for low levels of magnetic field, a tensile stress increases the magnetic susceptibility represented by $d M /\left.d H\right|_{\sigma}$ slope, whereas a compression decreases it. This effect is inverse at high magnetic field levels by Villari effect, and the modeling reproduces it fairly. A cycle-to-cycle comparison is however not possible without taking into account the effective quantity of magnetic material.

\subsection{Piezomagnetic hysteresis}

As explained in Section 4.2, the modeling of the piezomagnetic behavior proposed in this work is inspired from the description of the hysteretic irreversibility given by the Hauser's model. The definition of the irreversible stress 

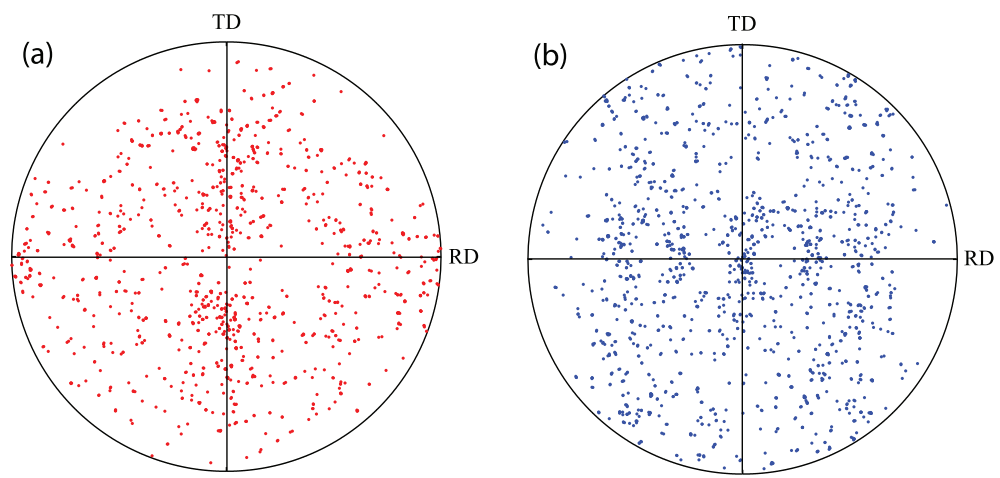

Fig. 6. Poles figures of the material: (a) $\langle 100\rangle$ poles; (b) $\langle 111\rangle$ poles. RD: rolling direction; TD: transverse direction.

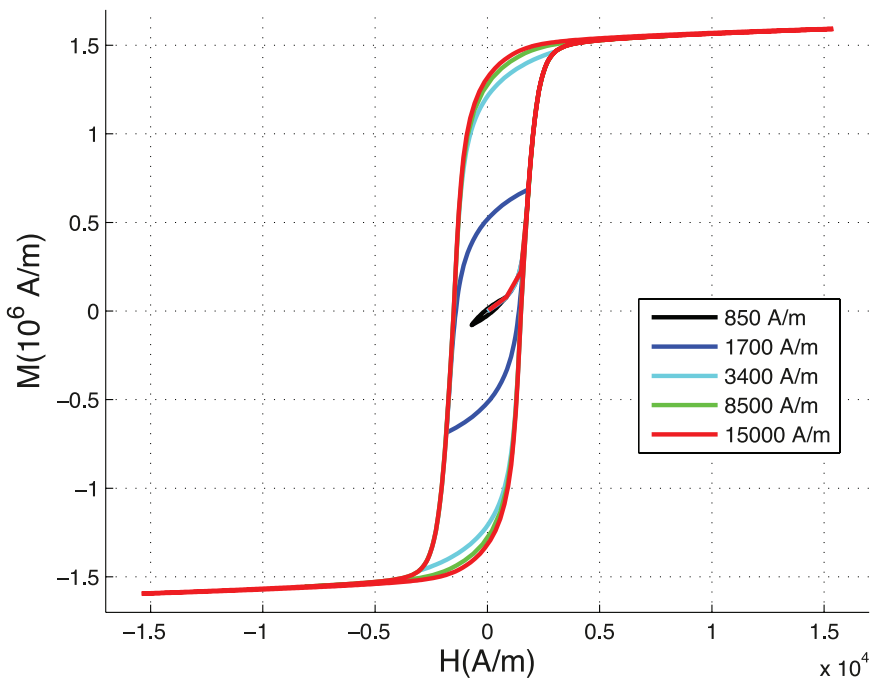

Fig. 7. Modeling results: evolution of the hysteretic cycles with the applied magnetic field $M=f(H, \sigma=0 \mathrm{MPa})$.

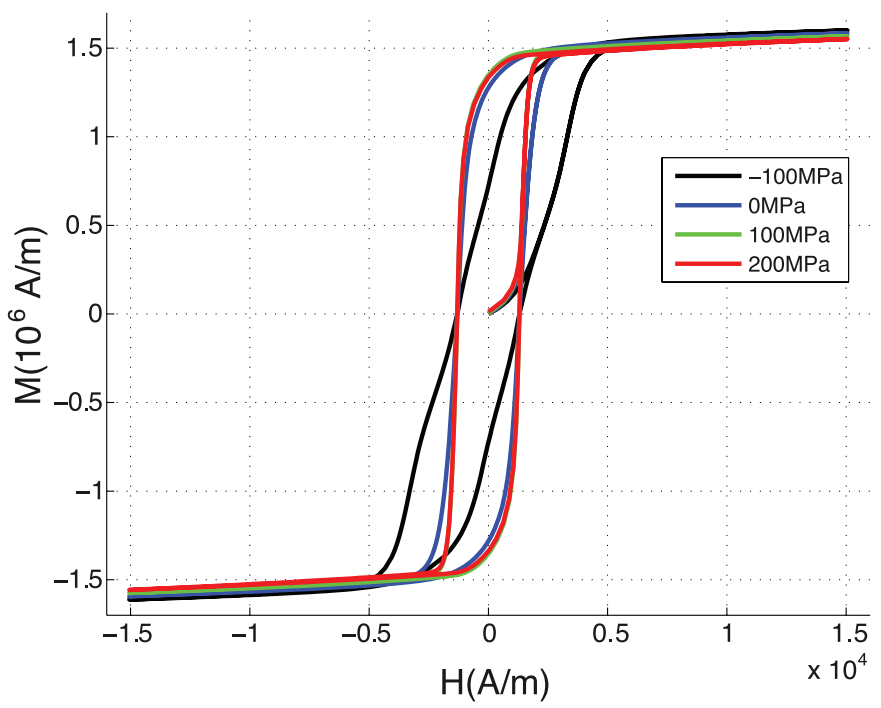

Fig. 8. Modeling results: hysteretic cycles under different mechanical loading conditions $M=f(H, \sigma=$ cte $)$.

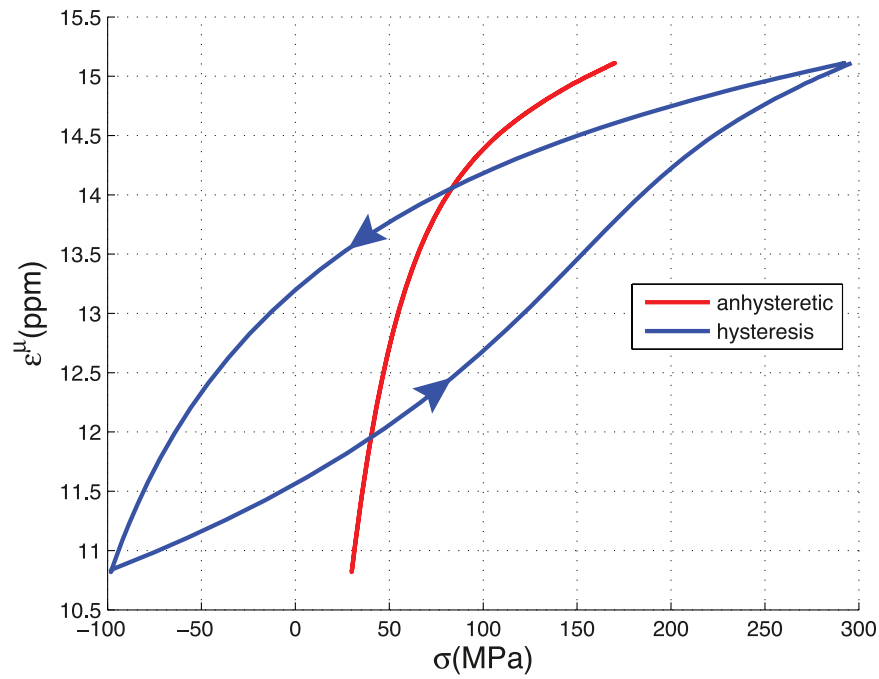

Fig. 9. Modeling results: stress-induced magnetostriction vs. stress cycle.

allows the modeling of the stress-induced magnetostriction. Figure 9 represents this cycle, representative of a mechanical dissipation. ${ }^{2}$ This cycle, drawn for pure iron, can be hardly obtained experimentally because magnetostrictive deformations are very low compared to elastic deformation.

Thanks to this modeling strategy we succeed to reproduce piezomagnetic irreversibility. The piezomagnetic cycles are calculated for different magnetic field levels $H_{\text {stat }}(850 \mathrm{~A} / \mathrm{m}, 1700 \mathrm{~A} / \mathrm{m}, 3400 \mathrm{~A} / \mathrm{m}, 8500 \mathrm{~A} / \mathrm{m}$, $15000 \mathrm{~A} / \mathrm{m}$ ) by plotting the magnetization as function of total stress $\left(\sigma=\sigma_{\text {rev }}+\sigma_{\text {irr }}\right)$ as shown in Figure 10. This result is given for pure iron. The mechanical loading used for modeling is a triangular stress waveform with a frequency of $f_{\text {mec }}=0.5 \mathrm{~Hz}$. The mechanical loading amplitude is not perfectly mastered since the component $\sigma_{\text {irr }}$ changes its value at each step of calculation. The cycles reproduced by the model show less variations and new phenomena such as the presence of cross points close to the Villari reversal points (Fig. 10), which has been

\footnotetext{
${ }^{2}$ The dissipation can be estimated at $320 \mathrm{~J} / \mathrm{m}^{3}$ per cycle. This value is much lower than the dissipation measured during a magnetic cycle that is about $9300 \mathrm{~J} / \mathrm{m}^{3}$ for a major cycle.
} 


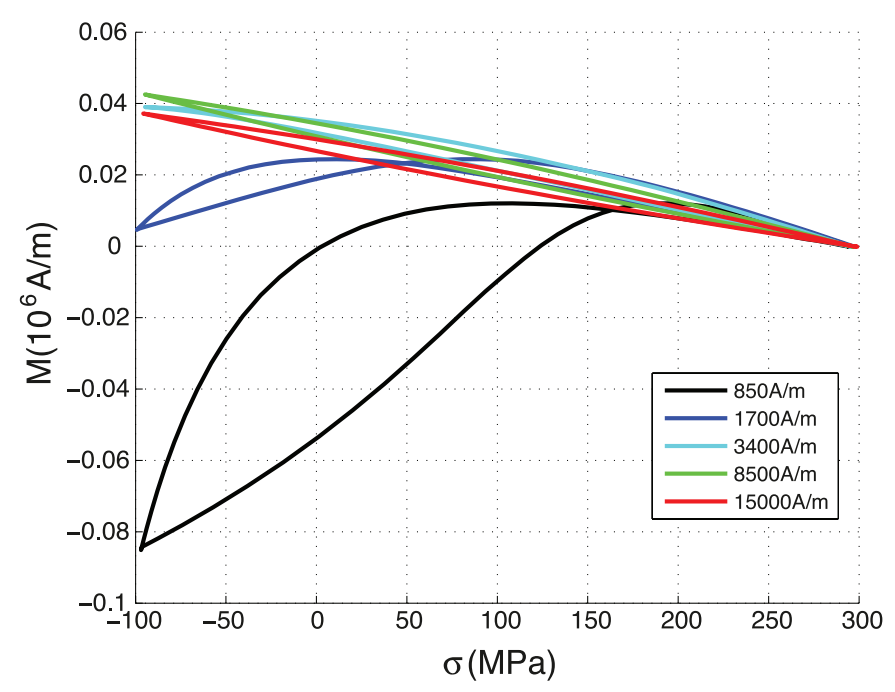

Fig. 10. Modeling results: evolution of the piezomagnetic cycles with the magnetic field level $M=f(H=$ cte, $\sigma)$.

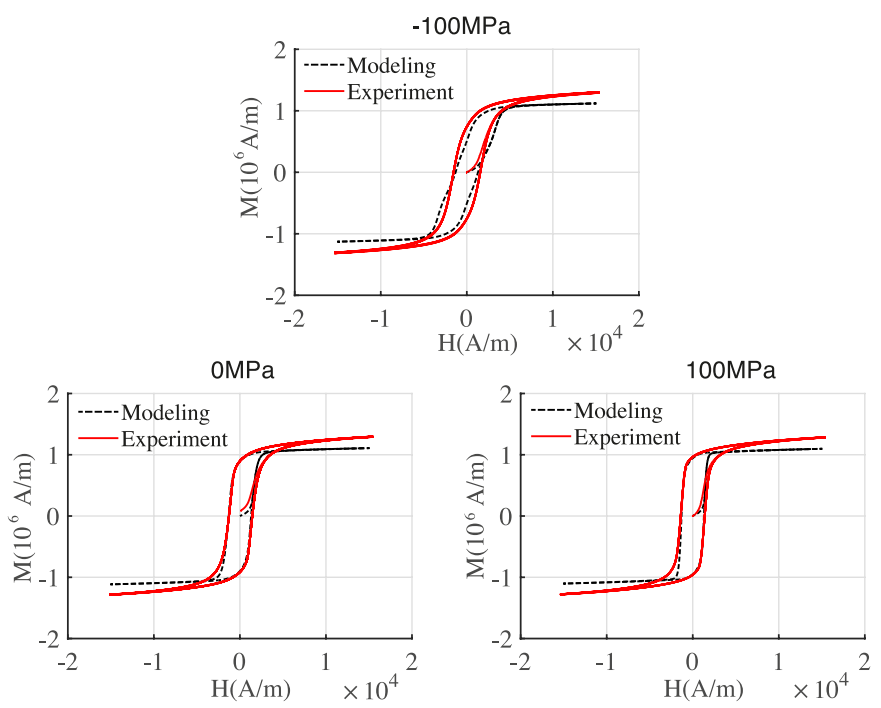

Fig. 11. Experimental measurements and modeling results under different mechanical loadings.

observed for other steels [5]. Nevertheless, a good general agreement with the experimental observations is obtained. For comparison of magnetization levels, the volume fraction of effective ferromagnetic material must be taken into account.

\subsection{Comparison between experimental measurements and modeling results}

The material is supposed to be composed of about $70 \%$ pure ferrite and that martensite brings non magnetic contribution to the magnetization. Figure 11 shows a comparison between the experimental and modeled magnetic behavior of the material under uniaxial stress $(0 \mathrm{MPa}$, $+100 \mathrm{MPa}$ and $-100 \mathrm{MPa})$. A good general agreement is observed. The model tends to underestimate the magnetization of the material for a given magnetic field, especially

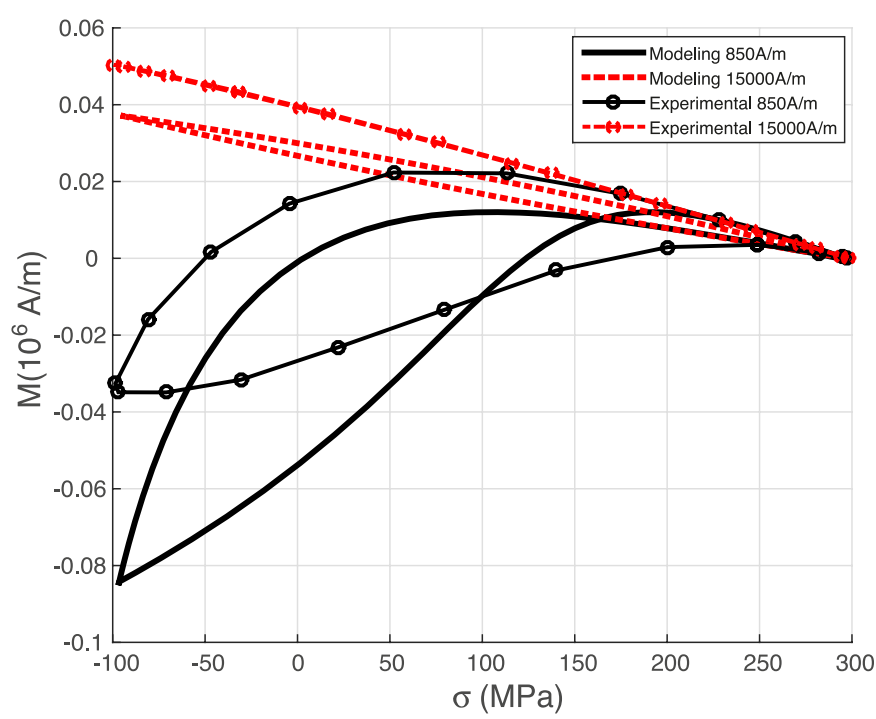

Fig. 12. Piezomagnetic behavior measured and calculated by the model.

at the magnetic saturation. Contribution of martensite is probably missing.

Figure 12 presents the measured and predicted piezomagnetic loops under static magnetic field. The model succeeds to reproduce the effect of the external magnetic field on the piezomagnetic behavior. Some peculiar experimental behaviors such as the change of the sign of the $d M /\left.d \sigma\right|_{H}$ slope and the decreasing of the dissipation are well rendered by the model. The contribution of martensite does not seem to be missing here. The amplitude of the modeled low-field cycle is even greater than the amplitude observed during the experiment.

\section{Conclusion}

The subject of this work was the study of the hysteretic and piezomagnetic behavior of a dual-phase steel. An experimental protocol was first presented. Hysteretic and piezomagnetic measurements were then performed under different loading (mechanical and magnetic) conditions. Experimental results are consistent with former results performed with low carbon steels. They highlight the effect of uniaxial stress and the magnetic field level on the magnetization of the material. A multiscale modeling has been used to calculate both the hysteretic and piezomagnetic behavior. Comparisons have been made between the prediction of modeled and measured results. The multiscale model seems able to describe correctly the effect of an applied stress and of an external magnetic field on the magnetization. Improvements are expected by taking the magnetic behavior of martensite phase into account. However, some shortcomings of the model are noticed: the magnetic and mechanical stress are considered homogeneous at the RVE scale and the behavior is only seen through the configuration of magnetic domains, neglecting some boundary and interface effects. Applications to the development of novel non destructive testing procedures is foreseen. 


\section{Appendix A: Localization and homogenization}

The calculation of the Gibbs free energy density at the magnetic domains scale requires knowing the stress applied at the grain scale constitutive of the polycrystal. The numerical applications in Section 5 were carried out using a homogeneous stress hypothesis for simplicity: the local (grain $=g$ ) stress is corresponding to the applied stress at the macroscale, leading to:

$$
\boldsymbol{\sigma}_{g}=\boldsymbol{\sigma}
$$

It is however possible to propose other estimates. The most common are the homogeneous strain and self-consistent schemes.

\section{A.1 Homogeneous strain estimate}

The deformation is considered as homogeneous when the deformation of each crystal is corresponding to the macroscopic deformation, leading to:

$$
\varepsilon_{g}=\varepsilon
$$

By additivity of the deformations of elastic and magnetostrictive origin, one obtains:

$$
\varepsilon_{g}^{e}+\varepsilon_{g}^{\mu}=\varepsilon^{e}+\varepsilon^{\mu}
$$

Elastic deformations are related to stresses via the stiffness tensors:

$$
\mathbb{C}_{g}^{-1}: \boldsymbol{\sigma}_{g}+\varepsilon_{g}^{\mu}=\mathbb{C}^{-1}: \boldsymbol{\sigma}+\varepsilon^{\mu}
$$

Stress at the grain scale is therefore linked to the macroscopic stress through a more complex relationship than in a situation of homogeneous stress:

$$
\boldsymbol{\sigma}_{g}=\mathbb{C}_{g}: \mathbb{C}^{-1}: \boldsymbol{\sigma}+\mathbb{C}_{g}:\left(\varepsilon^{\mu}-\varepsilon_{g}^{\mu}\right)
$$

Fourth rank tensor $\mathbb{B}_{g}=\mathbb{C}_{g}: \mathbb{C}^{-1}$ is the so-called stress concentration operator.

This relation must be completed by an estimation of the macroscopic stiffness tensor $\mathbb{C}$ and an estimation of the macroscopic magnetostriction strain $\varepsilon^{\mu}$. By considering on the one hand the problem as purely elastic, the homogeneous deformation assumption leads to:

$$
\boldsymbol{\sigma}_{g}=\mathbb{C}_{g}: \mathbb{C}^{-1}: \boldsymbol{\sigma}
$$

The average stress is obtained by an averaging of the local stresses following:

$$
\left\langle\boldsymbol{\sigma}_{g}\right\rangle=\boldsymbol{\sigma}=<\mathbb{C}_{g}: \mathbb{C}^{-1}: \boldsymbol{\sigma}>=<\mathbb{C}_{g}>: \mathbb{C}^{-1}: \boldsymbol{\sigma}
$$

where $\langle.$.$\rangle figures out a volume averaging (\langle.\rangle=$. $\left.\frac{1}{V} \int_{V} \ldots d v\right)$. This relationship leads to:

$$
\left\langle\mathbb{C}_{g}\right\rangle: \mathbb{C}^{-1}=\mathbb{I}
$$

where $\mathbb{I}$ is the fourth-rank identity operator, leading to the homogeneous deformation estimate of the macroscopic stiffness tensor (denoted as Voigt estimate):

$$
\mathbb{C}=\left\langle\mathbb{C}_{g}\right\rangle
$$

By applying on the other hand the average operation to the localization equation (A.5), one gets:

$$
\left\langle\boldsymbol{\sigma}_{g}\right\rangle=\left\langle\mathbb{C}_{g}: \mathbb{C}^{-1}: \boldsymbol{\sigma}\right\rangle+\left\langle\mathbb{C}_{g}:\left(\varepsilon^{\mu}-\varepsilon_{g}^{\mu}\right)\right\rangle
$$

leading to:

$$
\left\langle\mathbb{C}_{g}:\left(\varepsilon^{\mu}-\varepsilon_{g}^{\mu}\right)\right\rangle=\mathbf{0}
$$

$$
\left\langle\mathbb{C}_{g}\right\rangle: \varepsilon^{\mu}=\left\langle\mathbb{C}_{g}: \varepsilon_{g}^{\mu}\right\rangle
$$

$$
\varepsilon^{\mu}=\left\langle\mathbb{C}_{g}\right\rangle^{-1}:\left\langle\mathbb{C}_{g}: \varepsilon_{g}^{\mu}\right\rangle
$$

$$
\varepsilon^{\mu}=\left\langle\mathbb{C}^{-1}: \mathbb{C}_{g}: \varepsilon_{g}^{\mu}\right\rangle=\left\langle{ }^{t} \mathbb{B}_{g}: \varepsilon_{g}^{\mu}\right\rangle
$$

The average macroscopic magnetostriction is obtained thanks to this equation where ${ }^{t} \mathbb{B}_{g}$ indicates the transpose of $\mathbb{B}_{g}$.

The homogeneous deformation hypothesis leads to a self consistent estimation of local stress $\boldsymbol{\sigma}_{g}$, since it depends on the local and macroscopic magnetostriction tensor that depend on the local stress through the constitutive law. The computation time is consequently significantly higher than by using the homogeneous stress hypothesis.

\section{A.2 Self consistent estimate}

Each grain is considered as an inclusion in the homogeneous medium equivalent to the polycrystal, so that the problem can be linked to the solution of the Eshelby inclusion problem [15]. Considering on the one hand a macroscopic applied stress $\boldsymbol{\sigma}$, a local deformation $\varepsilon_{g}$ and a macroscopic deformation $\varepsilon$, the stress at the grain scale is given by the Hill's relationship [16]:

$$
\boldsymbol{\sigma}_{g}=\boldsymbol{\sigma}+\mathbb{C}^{\star}:\left(\varepsilon-\varepsilon_{g}\right)
$$

where $\mathbb{C}^{\star}$ is the Hill's constraint operator defined by:

$$
\mathbb{C}^{\star}=\mathbb{C}_{0}:\left(\mathbb{S}^{E-1}-\mathbb{I}\right)
$$

$\mathbb{C}_{0}$ is the stiffness tensor of the equivalent medium, taken as the macroscopic stiffness tensor $\mathbb{C}$ in case of selfconsistent estimate. $\mathbb{S}^{E}$ is the so-called Eshelby tensor. $\mathbb{S}^{E}$ only depends on the single crystal elastic moduli and on the shape chosen for the inclusion. Elements concerning the calculation of this tensor can be found in [17]. For the applications considered in Appendix A.3 inclusions are taken spherical, assuming an isotropic distribution of the grains. 
By additivity of the deformations of elastic and magnetostrictive origin, the Hill relationship (A.15) transforms into:

$$
\boldsymbol{\sigma}_{g}=\boldsymbol{\sigma}+\mathbb{C}^{\star}:\left(\varepsilon^{e}+\varepsilon^{\mu}-\varepsilon_{g}^{e}-\varepsilon_{g}^{\mu}\right) .
$$

Elastic deformations are related to stresses via the stiffness tensors, so that:

$$
\boldsymbol{\sigma}_{g}=\boldsymbol{\sigma}+\mathbb{C}^{\star}:\left(\mathbb{C}^{-1}: \boldsymbol{\sigma}+\varepsilon^{\mu}-\mathbb{C}_{g}^{-1}: \boldsymbol{\sigma}_{g}-\varepsilon_{g}^{\mu}\right)
$$

or

$$
\left(\mathbb{C}^{\star}: \mathbb{C}_{g}^{-1}+\mathbb{I}\right): \sigma_{g}=\left(\mathbb{C}^{\star}: \mathbb{C}^{-1}+\mathbb{I}\right): \sigma+\mathbb{C}^{\star}:\left(\varepsilon^{\mu}-\varepsilon_{g}^{\mu}\right)
$$

and

$$
\begin{aligned}
\boldsymbol{\sigma}_{g}= & \left(\mathbb{C}^{\star}: \mathbb{C}_{g}^{-1}+\mathbb{I}\right)^{-1}:\left(\mathbb{C}^{\star}: \mathbb{C}^{-1}+\mathbb{I}\right): \boldsymbol{\sigma} \\
& +\left(\mathbb{C}^{\star}: \mathbb{C}_{g}^{-1}+\mathbb{I}\right)^{-1}: \mathbb{C}^{\star}:\left(\varepsilon^{\mu}-\varepsilon_{g}^{\mu}\right) \\
\boldsymbol{\sigma}_{g}= & \mathbb{C}_{g}:\left(\mathbb{C}_{g}+\mathbb{C}^{\star}\right)^{-1}:\left(\mathbb{C}+\mathbb{C}^{\star}\right): \mathbb{C}^{-1}: \boldsymbol{\sigma} \\
& +\mathbb{C}_{g}:\left(\mathbb{C}_{g}+\mathbb{C}^{\star}\right)^{-1}: \mathbb{C}^{\star}:\left(\varepsilon^{\mu}-\varepsilon_{g}^{\mu}\right) \\
& \boldsymbol{\sigma}_{g}=\mathbb{B}_{g}: \boldsymbol{\sigma}+\mathbb{C}_{g}^{a c c}:\left(\varepsilon^{\mu}-\varepsilon_{g}^{\mu}\right) .
\end{aligned}
$$

The definition of stress concentration tensor is modified into: $\mathbb{B}_{g}=\mathbb{C}_{g}:\left(\mathbb{C}_{g}+\mathbb{C}^{\star}\right)^{-1}:\left(\mathbb{C}+\mathbb{C}^{\star}\right): \mathbb{C}^{-1} \cdot \mathbb{C}_{g}^{a c c}$ denotes the accommodation stiffness tensor given by: $\mathbb{C}_{g}^{a c c}=\mathbb{C}_{g}$ : $\left(\mathbb{C}_{g}+\mathbb{C}^{\star}\right)^{-1}: \mathbb{C}^{\star}$.

Equation (A.22) allows the local stress as function of macroscopic stress, macroscopic magnetostriction and local magnetostriction to be obtained. As for homogeneous strain estimate, this relation must be completed by an estimation of the macroscopic stiffness tensor $\mathbb{C}$ and an estimation of the macroscopic magnetostriction strain $\varepsilon^{\mu}$. By considering on the one hand the problem as purely elastic, the local and global stress are related by:

$$
\boldsymbol{\sigma}_{g}=\mathbb{C}_{g}:\left(\mathbb{C}_{g}+\mathbb{C}^{\star}\right)^{-1}:\left(\mathbb{C}+\mathbb{C}^{\star}\right): \mathbb{C}^{-1}: \boldsymbol{\sigma} .
$$

The average stress is obtained by an averaging of the local stresses following:

$$
\begin{aligned}
\left\langle\boldsymbol{\sigma}_{g}\right\rangle & =\boldsymbol{\sigma}=\left\langle\mathbb{C}_{g}:\left(\mathbb{C}_{g}+\mathbb{C}^{\star}\right)^{-1}:\left(\mathbb{C}+\mathbb{C}^{\star}\right): \mathbb{C}^{-1}:\right. \\
\boldsymbol{\sigma}\rangle & =\left\langle\mathbb{C}_{g}:\left(\mathbb{C}_{g}+\mathbb{C}^{\star}\right)^{-1}:\left(\mathbb{C}+\mathbb{C}^{\star}\right)\right\rangle: \mathbb{C}^{-1}: \boldsymbol{\sigma} .
\end{aligned}
$$

This relationship leads to:

$$
\left\langle\mathbb{C}_{g}:\left(\mathbb{C}_{g}+\mathbb{C}^{\star}\right)^{-1}:\left(\mathbb{C}+\mathbb{C}^{\star}\right)\right\rangle: \mathbb{C}^{-1}=\mathbb{I}
$$

The Self-Consistent estimate of the macroscopic stiffness tensor is obtained:

$$
\mathbb{C}=\left\langle\mathbb{C}_{g}:\left(\mathbb{C}_{g}+\mathbb{C}^{\star}\right)^{-1}:\left(\mathbb{C}+\mathbb{C}^{\star}\right)\right\rangle .
$$

Table A.1. Stiffness constants of the iron single crystal - using Voigt notations [11].

\begin{tabular}{llll}
\hline Parameter & $C_{11}$ & $C_{12}$ & $C_{44}$ \\
\hline Value & 238 & 142 & 232 \\
Unit & $\mathrm{GPa}$ & $\mathrm{GPa}$ & $\mathrm{GPa}$ \\
\hline
\end{tabular}

It must be remarked that this equation is an implicit equation since the equivalent medium stiffness is taken as the macroscopic stiffness itself.

By applying on the other hand the average operation to the localization equation (A.22), one gets:

$$
\begin{aligned}
\left\langle\boldsymbol{\sigma}_{g}\right\rangle= & \left.\left\langle\mathbb{C}_{g}:\left(\mathbb{C}_{g}+\mathbb{C}^{\star}\right)^{-1}:\left(\mathbb{C}+\mathbb{C}^{\star}\right)\right\rangle: \mathbb{C}^{-1}: \boldsymbol{\sigma}\right\rangle \\
& +\left\langle\mathbb{C}_{g}:\left(\mathbb{C}_{g}+\mathbb{C}^{\star}\right)^{-1}: \mathbb{C}^{\star}:\left(\varepsilon^{\mu}-\varepsilon_{g}^{\mu}\right)\right\rangle
\end{aligned}
$$

leading to:

$$
\begin{gathered}
\left\langle\mathbb{C}_{g}:\left(\mathbb{C}_{g}+\mathbb{C}^{\star}\right)^{-1}: \mathbb{C}^{\star}:\left(\varepsilon^{\mu}-\varepsilon_{g}^{\mu}\right)\right\rangle=\mathbf{0} \\
\left\langle\mathbb{C}_{g}:\left(\mathbb{C}_{g}+\mathbb{C}^{\star}\right)^{-1}: \mathbb{C}^{\star}\right\rangle: \varepsilon^{\mu} \\
=\left\langle\mathbb{C}_{g}:\left(\mathbb{C}_{g}+\mathbb{C}^{\star}\right)^{-1}: \mathbb{C}^{\star}: \varepsilon_{g}^{\mu}\right\rangle \\
\mathbb{C}:\left(\mathbb{C}+\mathbb{C}^{\star}\right)^{-1}: \mathbb{C}^{\star}: \varepsilon^{\mu}=\left\langle\mathbb{C}_{g}:\left(\mathbb{C}_{g}+\mathbb{C}^{\star}\right)^{-1}: \mathbb{C}^{\star}: \varepsilon_{g}^{\mu}\right\rangle \\
\varepsilon^{\mu}=\left\langle\mathbb{C}^{-1}:\left(\mathbb{C}+\mathbb{C}^{\star}\right):\left(\mathbb{C}_{g}+\mathbb{C}^{\star}\right)^{-1}: \mathbb{C}_{g}: \varepsilon_{g}^{\mu}\right\rangle \\
=\left\langle\mathbb{B}_{g}: \varepsilon_{g}^{\mu}\right\rangle .
\end{gathered}
$$

A self-consistent equation is of course obtained where the local and macroscopic magnetostriction tensors depend on the local stress through the constitutive law. The computation time is consequently significantly higher than by using the homogeneous stress hypothesis but close to the computation time observed with the homogeneous strain hypothesis.

\section{A.3 Illustrations}

The two schemes have been applied for the calculation of the macroscopic magnetostriction strain and magnetization during a piezomagnetic cycle. This calculation requires the use of the pure iron stiffness constants as three more parameters. They are given in Table A.1.

Figures A.1a and A.1b illustrate the corresponding results compared to the homogeneous stress estimate already reported in the main text.

It can be observed that homogeneous deformation condition leads to a reduced magnitude of magnetostriction and magnetization comparing to the homogeneous stress condition. This result was awaited since homogeneous deformation usually enhances the mechanical contrast between grains. Stress always opposes to the free deformations. The self-consistent estimate leads to an intermediate behavior between the two previous estimates. 

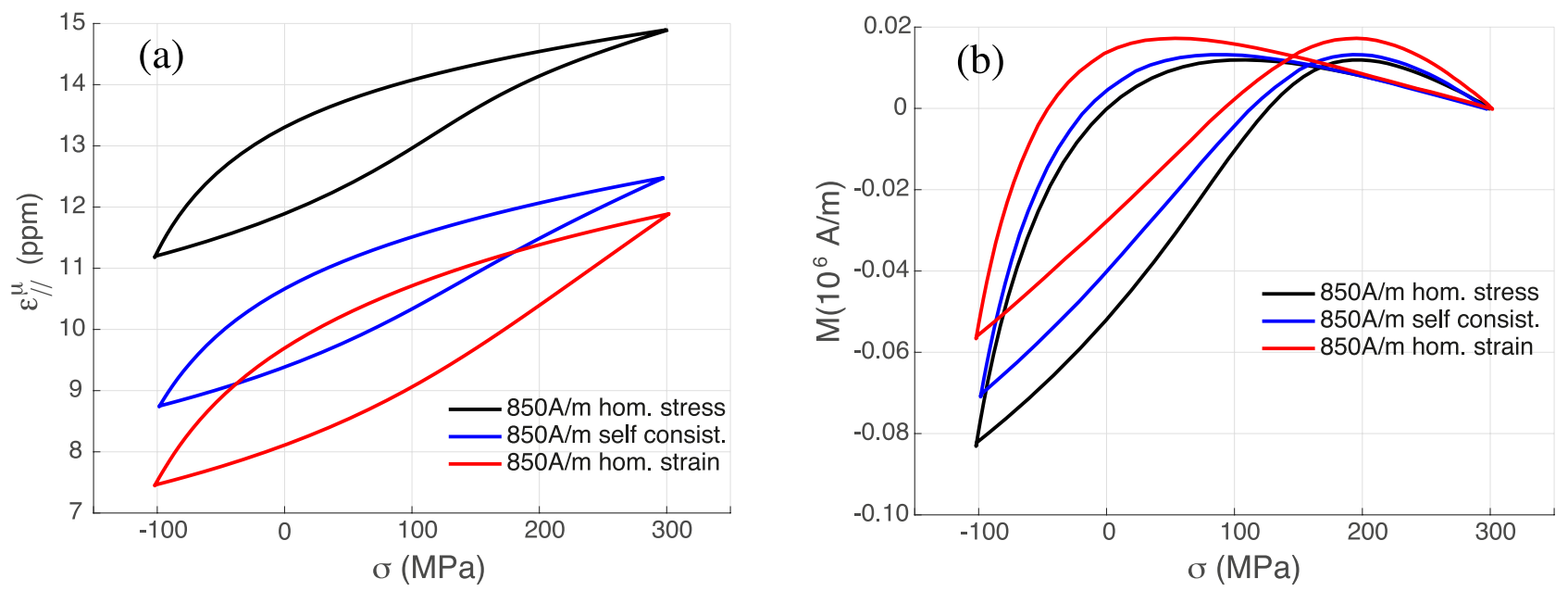

Fig. A.1. Comparison between homogeneous stress, homogeneous strain and self-consistent estimates: (a) stress-induced magnetostriction vs. stress cycles; (b) piezomagnetic cycles.

\section{References}

[1] E. Villari, Change of magnetization by tension and by electric current, Ann. Phys. Chem. 126, 87-122 (1865)

[2] R.M. Bozorth, Ferromagnetism, Van Nostrand (Ed.), Wiley IEEE Press, New York, 1951

[3] J. Dumont-Fillon, Mesures, Analyses/Contôle non destructif, Techniques de l'ingénieur, 1996

[4] L. Lollioz, S. Pattofatto, O. Hubert, Application of piezomagnetism for the measurement of stress during an impact, J. Elect. Eng. 57, 15-20 (2006)

[5] O. Hubert, K.J. Rizzo, Anhysteretic and dynamic piezomagnetic behavior of a low carbon steel, J. Mag. Mag. Mater. 320, 979-982 (2008)

[6] S. Bao, T. Erber, S.A. Guralnick, W.L. Jin, Fatigue, Magnetic and Mechanical Hysteresis, Strain 47, 372-381 (2011)

[7] K.J. Rizzo, O. Hubert, L. Daniel, A multiscale model for piezomagnetic behavior, Eur. J. Electric. Eng. 12, 525-540 (2009)

[8] F.S. Mballa-Mballa, O. Hubert, S. Lazreg, P. Meilland, Multidomain modelling of the magneto-mechanical behaviour of dual-phase steels, 18th WCNDT - World Conference on Nondestructive Testing. 16-20 April 2012, Durban (South Africa)

[9] O. Hubert, S. Lazreg, Two phase modeling of the influence of plastic strain on the magnetic and magnetostrictive behaviors of ferromagnetic materials, J. Mag. Mag. Mater. 424, 421-442 (2017)

[10] O. Hubert, Multiscale magneto-elastic modeling of magnetic materials including isotropic second order stress effect, J. Magn. Magn. Mater. 491, 1-16 (2019)

[11] L. Daniel, O. Hubert, N. Buiron, R. Billardon, Reversible magneto-elastic behavior: a multiscale approach, J. Mech. Phys. Solids 56, 1018-1042 (2008)

[12] L. Daniel, M. Rekik, O. Hubert, A multiscale model for magneto-elastic behaviour including hysteresis effects, Arch. Appl. Mech. 84, 1307-1323 (2014)

[13] X. Chang, K. Lavernhe, O. Hubert, Stochastic multiscale modeling of the thermomechanical behavior of polycrystalline shape memory alloys, Mech. Mater. 144, 1-27 (2020)

[14] H. Hauser, Energetic model of ferromagnetic hysteresis: Isotropic magnetization, J. Appl. Phys. 96, 2753-2767 (2004)

[15] J.D. Eshelby, The determination of the elastic field of an ellipsoidal inclusion, and related problems, Proc. R. Soc. London, Ser. A 241, 376-396 (1957)

[16] R. Hill, Continuum micro-mechanics of elastoplastic polycrystals, J. Mech. Phys. Solids 13, 89-101 (1965)

[17] T. Mura, Micromechanics of Defects in Solids, Martinus Nijhoff Publishers, Dordrecht, MA, 1982

Cite this article as: A. Ouaddi, O. Hubert, J. Furtado, D. Gary, S. Depeyre, Piezomagnetic behavior: experimental observations and multiscale modeling, Mechanics \& Industry 20, 810 (2019) 